\title{
In-Process Simulation of Wire Electrode Displacement in WEDM using Optical Sensor*
}

\author{
Kazuki SHIBATA $^{* *}$ and Masanori KUNIEDA $* * *$
}

\begin{abstract}
During wire electrical discharge machining, the wire electrode is deflected by various forces like discharge reaction force. Since the generated deflection and vibration affect processing accuracy, it is necessary to accurately control the wire behavior during machining in real time. Thus in this research, the authors proposed a real-time simulation method to obtain data on the three dimensional wire behavior. The discharge reaction force was obtained from the inverse problem method in which the discharge reaction force was determined so that the two dimensional displacement of the wire electrode measured by an optical sensor coincides with the calculated displacement. A prototype of the simulation system was made, and the change of the wire electrode behavior was measured during rough machining with increasing cutting depth. In addition, the influence of the fluid flow on the wire behavior, which is not negligibly small compared to the discharge reaction force, was investigated using the optical sensor.
\end{abstract}

Key words: wire electrical discharge machining (WEDM), wire electrode vibration, optical sensor, machining accuracy, in-process simulation, discharge reaction force.

\section{Introduction}

There are four dominant forces acting on the wire electrode during wire electrical discharge machining (WEDM): discharge reaction force, electrostatic force, electromagnetic force, and the force generated by the flow of machining fluid ${ }^{1}$. Due to these forces, the wire is bent and oscillated, and its behavior is complicated and difficult to control accurately ${ }^{1)-3}$. Therefore, simulation of the wire electrode behavior is necessary to realize high accuracy machining. The workpiece shape can be obtained from the envelope surface of the deflected wire. To calculate the wire behavior however, the four forces must be known. When Han et $\mathrm{al}^{2}$ simulated the wire behavior, they used the electrostatic force obtained from analysis. However, the discharge reaction force was obtained from the inverse problem method by comparing the simulated workpiece shape with the measured one. Tomura et $\mathrm{al}^{3)}$ simulated the wire behavior more accurately by taking the electromagnetic force into account. However, the method for calculating the discharge reaction force has not yet been developed. Moreover, the discharge reaction force may vary depending on the materials used for the wire electrode and workpiece ${ }^{4}$, thickness of the workpiece, and the cutting depth even

* Received on February 28, 2019. Accepted on May 16, 2019.

“ Student member, Department of Precision Engineering, The University of Tokyo (7-3-1, Hongo, Bunkyo-ku, Tokyo, 1138656, JAPAN)

*** Full member, Department of Precision Engineering, The University of Tokyo (7-3-1, Hongo, Bunkyo-ku, Tokyo, 1138656, JAPAN) under the same pulse conditions. Hence, the discharge reaction force obtained from the inverse problem under a specific machining condition cannot be used in other situations. Furthermore, the influence of the jet of the machining fluid on the wire behavior ${ }^{5), 6)}$ has not been investigated.

In this study, a pair of sensors developed by Nishikawa et al. ${ }^{7)}$ to measure the wire displacement during machining without interfering the process were installed in a plane perpendicular to the wire axis, and the two dimensional wire displacement in the plane was measured. Then, a real time simulation system to calculate the three dimensional deflection of the wire was developed. This system uses the discharge reaction force, which changes in the process, obtained by the inverse problem by comparing the two dimensional wire displacement being measured in the process with the calculated one. Using this system developed, the three dimensional wire behavior was measured with increasing the cutting depth in rough machining, and then the measured results were compared with the workpiece shape measured after machining. Moreover, to separate the influence of the flow field from the apparent discharge reaction force obtained, only the wire displacement due to the flow field is measured.

\section{Principle of optical wire displacement sensor ${ }^{7)}$}

A schematic diagram of the wire displacement sensor is shown in Fig. 1. The wire displacement sensor consists of a collimator lens to emit a parallel $\mathrm{He}$ - Ne laser beam of $0.48 \mathrm{~mm}$ in diameter, and a pair of plastic optical fibers with a core diameter of $0.235 \mathrm{~mm}$ and clad thickness of $7.5 \mu \mathrm{m}$. The plastic optical fibers contacting each other are aligned parallel to the laser beam and laid on a plane 


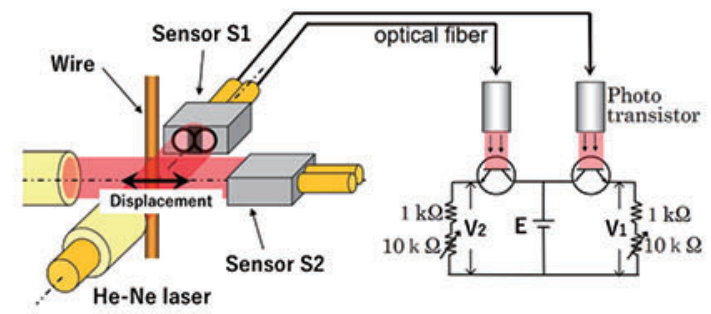

Fig. 1 Principle of wire displacement sensor.

perpendicular to the wire axis. The lights incident to the plastic fibers are guided to different phototransistors, and the light intensities are converted into voltages $V_{l}$ and $V_{2}$, respectively. When the wire shadows the laser beam, the silhouette of the wire is projected on the aperture of the optical fibers. The projection area depends on the wire position, thereby $V_{l}$ and $V_{2}$ are varied. By calibrating the relationship between the sensor outputs and wire displacement beforehand, the wire displacement can be obtained from the sensor outputs. In this research, we installed two sensors in two orthogonal axes, respectively, to enable measurement of the two-dimensional displacement of the wire in the plane perpendicular to the wire axis. The frequency characteristic of this sensor is about $2 \mathrm{kHz}$.

\section{Simulation model of WEDM}

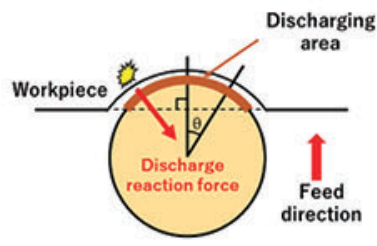

Fig. 2 Direction of discharge reaction force acting on wire.

To calculate the wire deflection, the wire was assumed to behave as a beam. $\mathrm{Han}^{2)}$ and Tomura ${ }^{3)}$ ignored the force generated by the flow of the machining fluid, and took into consideration of the discharge reaction force, electrostatic force, electromagnetic force, and collision of the wire with the workpiece. They also modelled the shape of the workpiece and calculated changes in the shape due to removal, where the point with shortest gap width was determined as the discharge spot. In this research however, for simplicity, the collision, electromagnetic force, electrostatic force, and force generated by the flow were neglected. Also, the changes in the shape of the workpiece due to machining were not considered. The discharge position in the axial direction was assumed to be distributed at random on the wire side surface facing the workpiece regardless of the gap width difference. It was also assumed that discharge occurs only on the circumference of the wire facing the cut surface of the workpiece, and that the discharge reaction force is directed from the discharge point to the center of the wire axis. The discharge probability per unit area on the wire circumference was assumed to be proportional to the volume of removal loaded to the unit area on the wire surface per unit time, that is, it was assumed to change in proportion to $\cos \theta$ in Fig. 2. The equation to solve the deflection of the wire can be expressed as,

$$
\begin{aligned}
& E I \frac{\partial^{4} x}{\partial z^{4}}-T \frac{\partial^{2} x}{\partial z^{2}}+\rho A \frac{\partial^{2} x}{\partial t^{2}}+c \frac{\partial x}{\partial t}=F_{x}(z, t) \\
& E I \frac{\partial^{4} y}{\partial z^{4}}-T \frac{\partial^{2} y}{\partial z^{2}}+\rho A \frac{\partial^{2} y}{\partial t^{2}}+c \frac{\partial y}{\partial t}=F_{y}(z, t)
\end{aligned}
$$

$\left[\begin{array}{ll}E: \text { Young's modulus } & I: \text { moment of inertia of area } \\ T \text { : wire tension } & \rho \text { : wire mass per unit length } \\ A: \text { cross sectional area } & c \text { : damping coefficient } \\ \left(F_{x}, F_{y}\right): \text { discharge reaction force }\end{array}\right]$

Here, the discharge reaction force $\left(F_{x}, F_{y}\right)$ and the damping coefficient $c$ are unknown. Therefore, the discharge reaction force was determined by the inverse problem method so that the time average of the wire axis coordinates measured by the experiment and the deflection of the wire at the position of the sensor in the simulation are equal. In the same way, $c$ was obtained by comparing the calculated and measured amplitudes of the wire vibration at the sensor position.

\section{Measurement of wire displacement at each depth of cut}

Using the wire displacement sensor and the simulation model described above, the behavior of the wire electrode was investigated while changing the cutting depth in rough-cutting.

First, using a brass wire electrode with a diameter of $0.2 \mathrm{~mm}$, a stainless workpiece with a thickness of $30 \mathrm{~mm}$ was rough-cut. The wire was fed in the direction perpendicular to the side of the workpiece with the wire tension of $8 \mathrm{~N}$. The coordinate system was defined as shown in Fig. 3, where the origin of $X$ coordinate is located on the path of the wire feed, and the origin of $Y$ is on the edge of the workpiece. Then, the wire behavior from 1s before the start of discharging to $9 \mathrm{~s}$ after discharging was measured, and at the same time, the displacement of the wire guide and discharge frequency were also measured. The change in the discharge frequency was obtained from the discharge current waveform measured. The optical sensors were attached at the position $15 \mathrm{~mm}$ beneath the upper wire guide as shown in Fig. 4. Since the sensor is fixed to the upper wire guide, the wire displacement relative to the wire guide can be measured.

Next, to measure the shape of the kerf made in the workpiece, discharge was terminated when the wire guide was positioned at (1) $-0.1 \mathrm{~mm}$, (2) $0 \mathrm{~mm}$, (3) $0.1 \mathrm{~mm}$, and (4) $0.2 \mathrm{~mm}$ in the $Y$ direction. Then the profile of the kerf on the upper surface of the workpiece was measured using a high magnification measuring microscope. The machining conditions are shown in Table 1. 


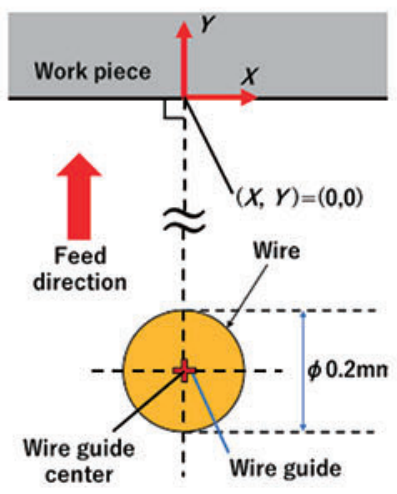

Fig. 3 Measurement of wire displacement in rough-cutting.

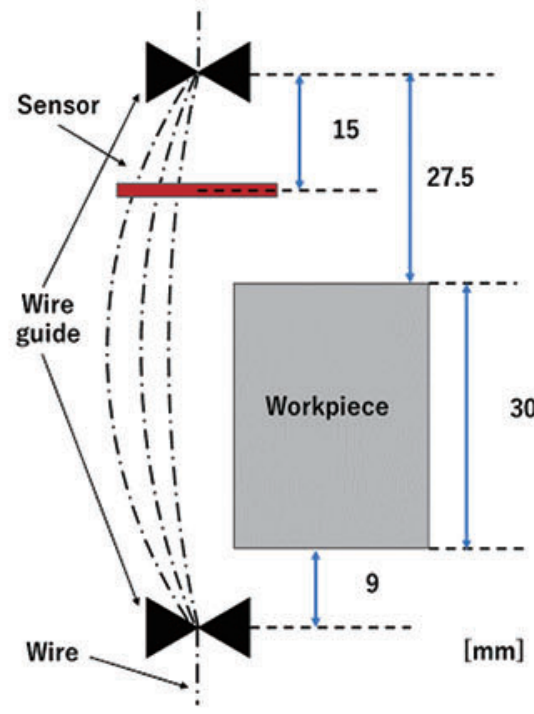

Fig. 4 Sensor position.

Table 1 Machining conditions.

\begin{tabular}{c|c}
\hline Discharge current & $160 \mathrm{~A}$ \\
\hline Discharge duration & $2 \mu \mathrm{s}$ \\
\hline Discharge interval & $14 \mu \mathrm{s}$ \\
\hline Servo voltage & $40 \mathrm{~V}$ \\
\hline Open voltage & $80 \mathrm{~V}$ \\
\hline Wire & $\phi 0.2 \mathrm{~mm}$, brass \\
\hline Wire tension & $8 \mathrm{~N}$ \\
\hline Wire running speed & $8 \mathrm{~m} / \mathrm{min}$ \\
\hline Flow rate of working fluid & $15 \mathrm{~L} / \mathrm{min}$ \\
\hline
\end{tabular}

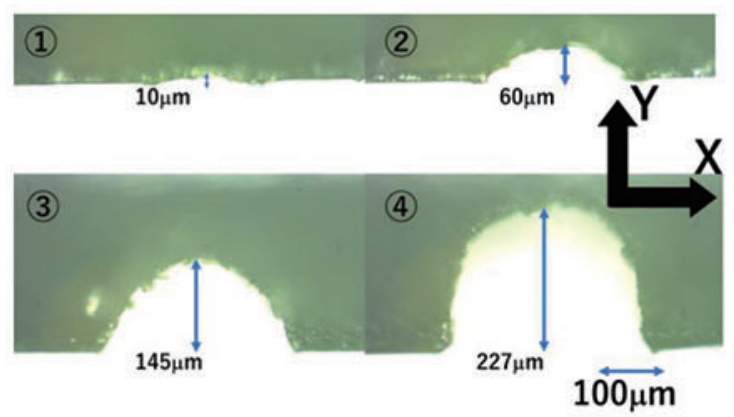

Fig. 5 Profile of kerf with different depth of cut.

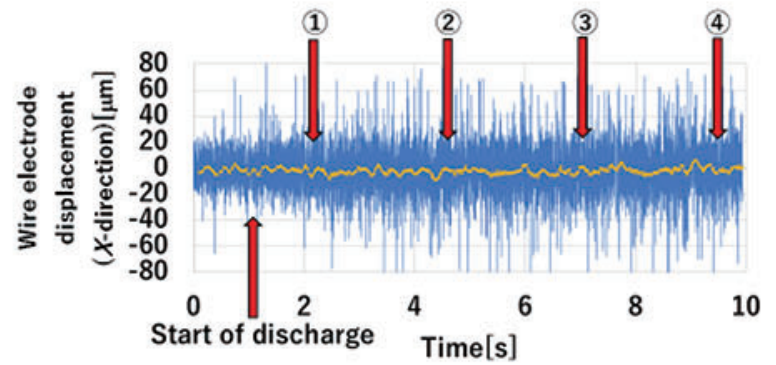

Fig. 6 Measured wire electrode displacement in $X$ direction.

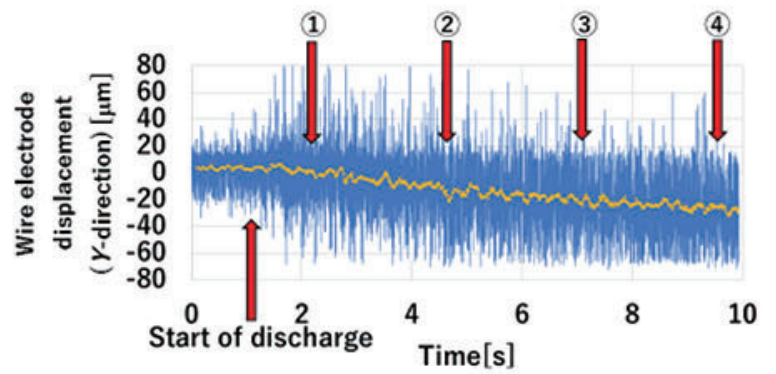

Fig. 7 Measured wire electrode displacement in $Y$ direction.

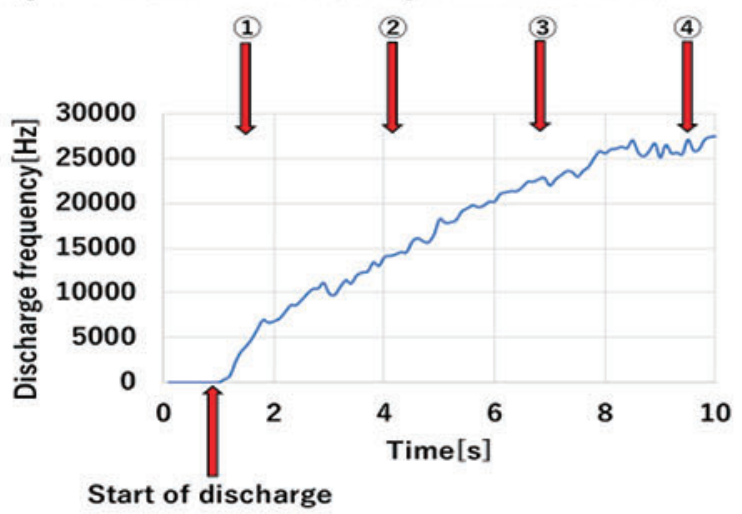

Fig. 8 Change in discharge frequency measured.

The profiles of the kerf on the upper surface of the workpiece are shown in Fig. 5, and the displacements in the $X$ and $Y$ directions are shown in Figs. 6 and 7, respectively. The yellow lines in Fig. 6 and 7 are the moving averages for $0.1 \mathrm{~s}$, and (1), (2), (3), and (4) indicate the situations where $Y$-coordinate of the wire guide was (1)- $0.1 \mathrm{~mm}$, (2) $0 \mathrm{~mm}$, (3) $0.1 \mathrm{~mm}$, and (4) $0.2 \mathrm{~mm}$, respectively. Fi gure 8 shows the change in the discharge frequency.

From the experimental results, it can be seen that the amplitude in both the $X$ and $Y$ directions increased after discharge started. The vibration in the $Y$ direction was larger than that in the $X$ direction because the discharge reaction force is acting on the wire mostly in the $Y$ direction, and the vibration is more restricted by the side surfaces in the kerf. It can also be seen that the wire deflection in the $Y$ direction gradually increased as the cutting depth increased.

This is due to the increase in the discharge frequency as shown in Fig. 8. Another reason is that during the formation of the kerf, the pressure of the bubbles generated by discharge increases in a narrow space, resulting in the increase in the discharge reaction force applied to the wire. 


\section{Simulation results}

The simulation of each situation from (1) to (4) was performed using the measurement results of the two dimensional wire displacement. As described above, the discharge reaction force and the damping coefficient $c$ were identified using the inverse problem method at each depth of cut from (1) to (4) so that the deflection and vibration of the wire in the $Y$ direction at the height where the sensor is attached are equal between the simulation and the experimental results. The identified damping coefficient was constantly $0.9 \mathrm{~N} \cdot \mathrm{s} / \mathrm{m}$ in all situations, while the impulse by the discharge reaction force per single discharge increased with the depth of cut as shown in Fig. 9.

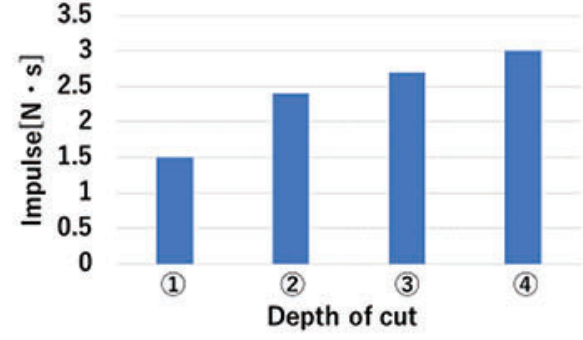

Fig. 9 Change in discharge reaction force per single discharge with depth of cut.
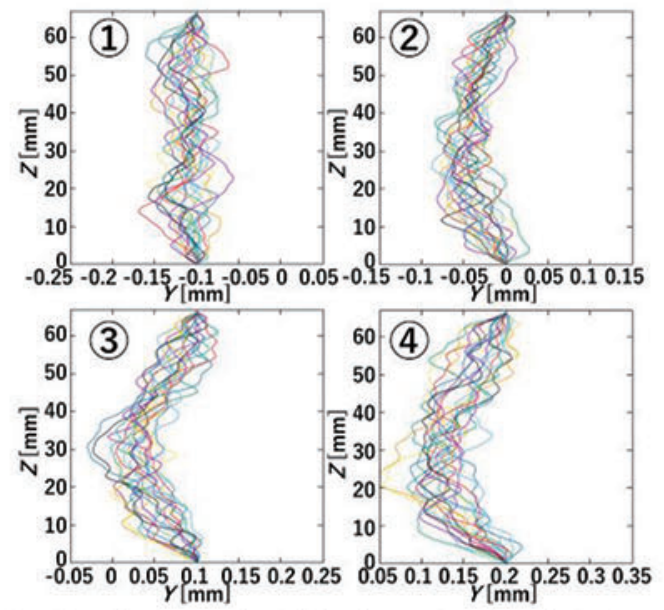

Fig. 10 Simulated wire deflection projected on $Y Z$ plane.
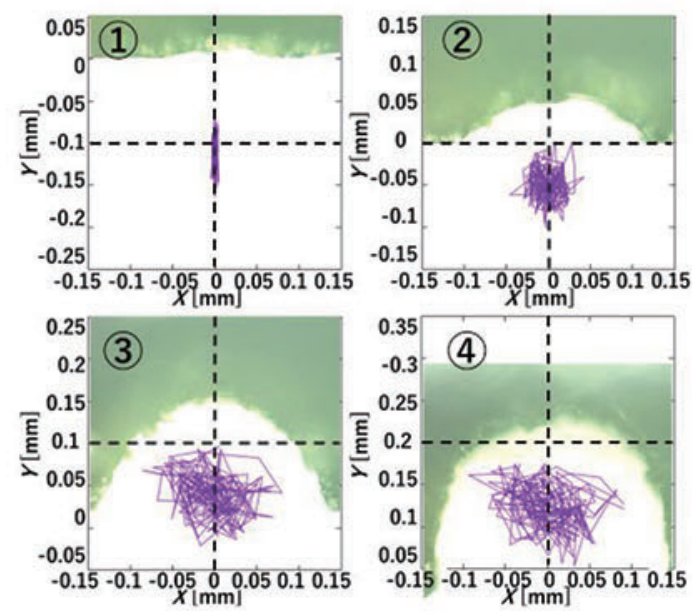

Fig. 11 Trajectory of wire center calculated on top surface of workpiece.
This is probably because the expansion of the bubble generated by discharge becomes more suppressed by the fluid flow resistance in the narrow space of the kerf with increasing depth of cut as mentioned in the previous section. Figure 10 shows the displacement of the wire projected on the $Y Z$ plane simulated at each situation (1) to (4), using the above identified values. Figure 11 shows the trajectory of the center of the wire calculated at the top surface of the workpiece together with the profile of the kerf photographed on the same surface. In this figure, the photograph of the profile of the kerf is shown on this top surface, and the wire guide center is shown by the intersection of the broken lines.

From Fig. 10, it can be confirmed that the wire is bent more significantly with increasing depth of cut. From Fig. 11, it can also be confirmed that the vibration in the $Y$ direction is larger than that in the $X$ direction at the beginning, while it becomes more or less the same after cutting progresses. This is because discharge points are being distributed around the wire as the depth of cut increases. These results indicate that the measurement of the wire displacement in combination with the simulation realizes real-time measurement of the discharge reaction force and $3 \mathrm{D}$ wire behavior.

Considering the diameter of the wire, Fig. 11 shows that the wire is interfering with the workpiece due to its diameter. This is because neither the collision of wire with the workpiece nor the force due to the fluid flow is taken into consideration in the simulation.

\section{Effect of flushing fluid jet on wire deflection and vibration}

In this simulation, since it was assumed that the force acting on the wire is the discharge reaction force only, the obtained results were not accurate. Especially, the influence of the force due to the flow field on the wire deflection and vibration cannot be ignored for

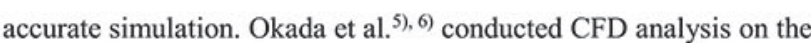
wire deflection caused by the jet. However, they did not investigate the influence of the fluid flow on the wire vibration. Its degree of influence on the wire deflection and vibration during the actual discharge process has not been compared with the influence of the discharge reaction force yet.

Thus, to investigate the influence of the fluid force on both the wire deflection and vibration, the wire displacement was measured during rough-cut using the sensor developed. Under the same machining conditions used in Section 4, the workpiece was cut as shown in Fig. 12 , and the wire behaviors when the wire guide center was being positioned at (1) to (6) were measured. For each position, cutting was repeated from the same start point. When the wire guide center reached (1) to (6), both discharge and wire feed were interrupted. Then, the displacement of the wire with and without the flushing jet was measured at each position, with the wire electrode wound at the wire running speed of $8 \mathrm{~m} / \mathrm{min}$. The measurement results are shown 
in Figs. 13 to 15. The deflection, defined as the average of the displacement, and the vibration amplitude, defined as the standard deviation of the displacement, in the $Y$ direction are shown in Fig. 16.

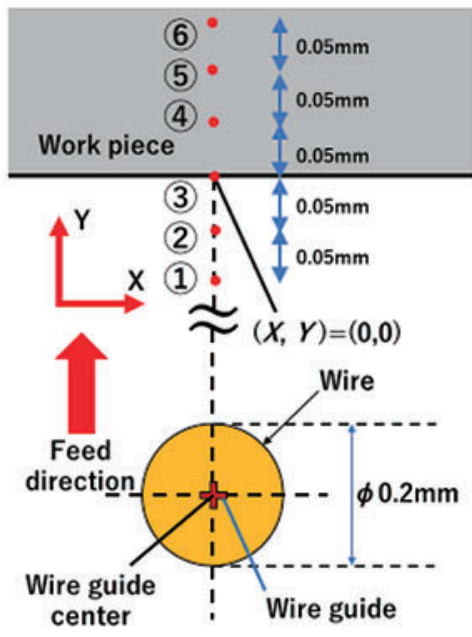

Fig. 12 Measurement of wire displacement due to fluid flow.

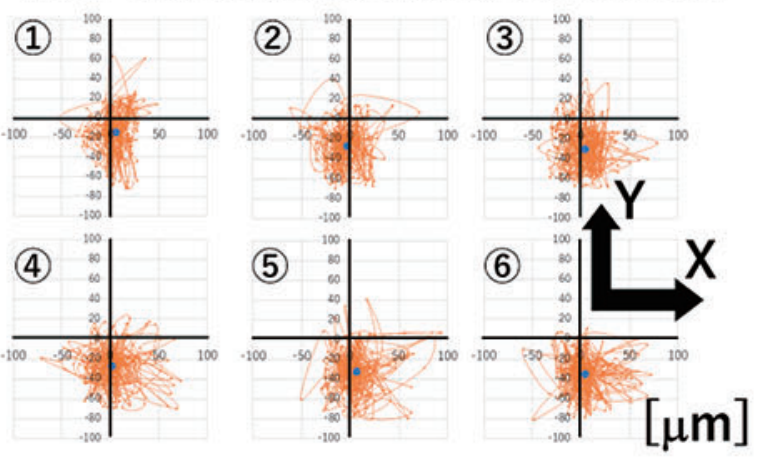

Fig. 13 Measurement results of wire behavior during machining.

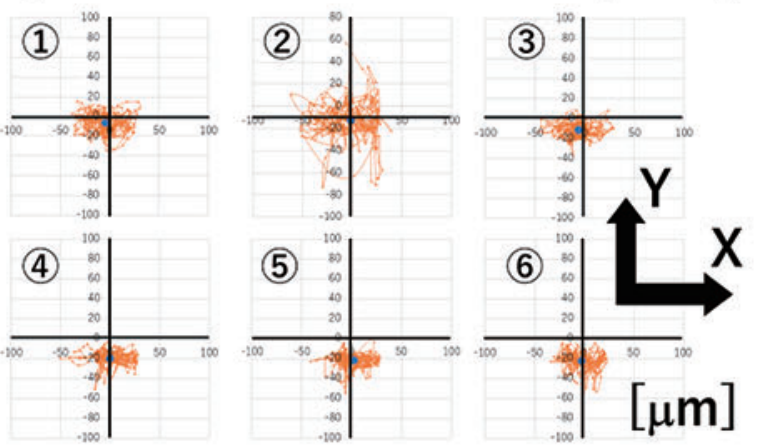

Fig. 14 Measurement results of wire behavior when machining is being interrupted, with jet flushing and wire running.

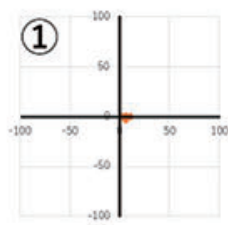

(4)
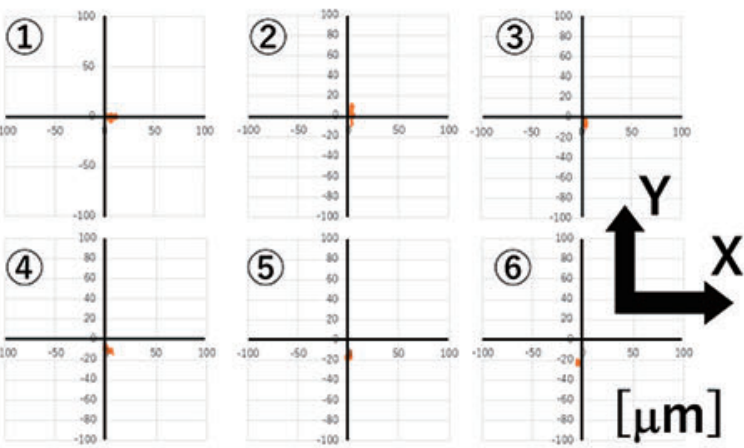

Fig. 15 Measurement results of wire behavior when machining is being interrupted, without jet flushing but with wire running.
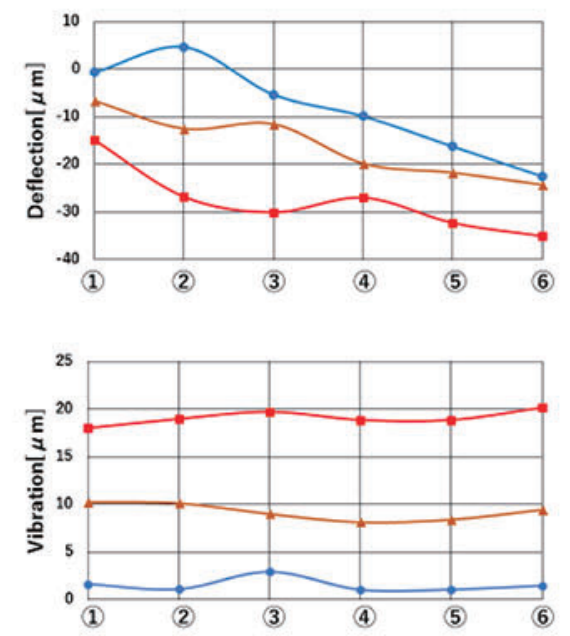

$\rightarrow-$ During machining

- Machining is interrupted, with jet flushing and wire running

- Machining is interrupted, without jet flushing, but with wire running

Fig. 16 Deflection and vibration amplitude in $Y$ direction.

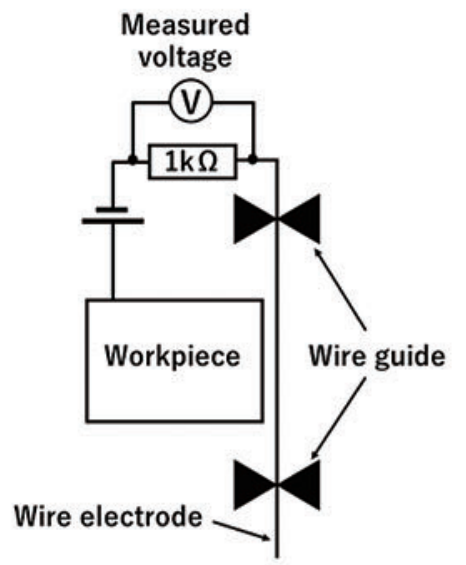

Fig. 17 Method to detect contact between wire and workpiece.

Figure 16 shows that when both machining and jet flushing are being interrupted but the wire is still running, the vibration of the wire is small. However, when the jet is supplied, the wire vibrates significantly and bends in the direction opposite to the feed direction. The vibration and deflection become larger during discharging. Nevertheless, the influence of the fluid force is not negligibly small.

In the previous section, Fig. 11 shows that the wire is making contact with the workpiece. Hence, it was investigated whether the wire and the workpiece are contacting each other when the wire was deflected by the jet. The electric feeder of the discharge circuit was disconnected from the table of the WEDM machine and a significantly low voltage of about $1 \mathrm{~V}$ was applied between the table and wire using a high resistance of $1 \mathrm{k} \Omega$ connected in a series to avoid the deflection of the wire due to discharge in the gap between the wire and workpiece as shown in Fig. 17. Then, the voltage drop in the resistor was measured using an oscilloscope. 


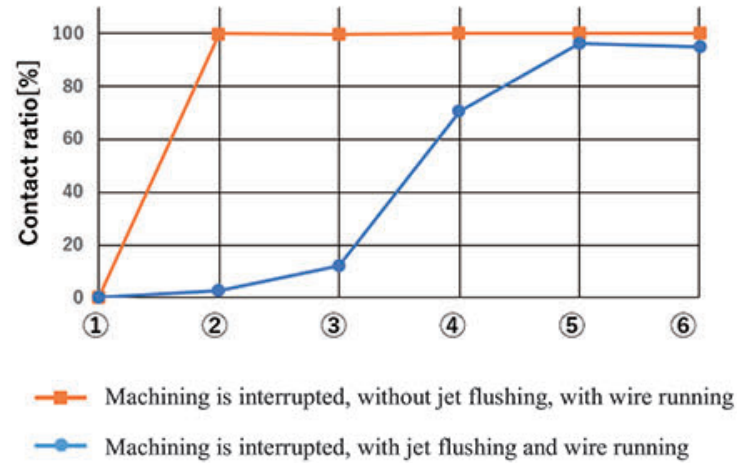

Fig. 18 Contact ratio between wire and workpiece.

When the wire and workpiece were in contact with each other the voltage was $1 \mathrm{~V}$, and when they were not in contact the voltage was approximately $0 \mathrm{~V}$. Thus, when the value was $0.1 \mathrm{~V}$ or less, it was judged that the wire and workpiece were not in contact with each other. In the situations (1) to (6), the voltage was measured for $10 \mathrm{~s}$ when machining was interrupted with the jet supplied, and when machining was interrupted without jet flushing. In both cases, the wire was running. The results are shown in Fig. 18.

It was found that in the situation of (2) or (3), the wire and the workpiece were not in contact with each other when the jet was supplied, but they were in contact when the jet was not supplied. This result indicates that the wire is bent not only due to the collision with the workpiece but also due to the influence of the fluid flow. This is presumably because a lift force is generated by the flow velocity which is faster on the other side of the workpiece than in the interelectrode gap.

According to the findings of these experiments, when the wire is near the workpiece, the jet deflects the wire toward the opposite side of the workpiece, and the wire is oscillated significantly by both the fluid force and discharge reaction force in rough-cutting.

\section{Conclusions}

1) A system to measure the two dimensional displacement of the wire electrode during machining was newly developed.

2) $2 \mathrm{D}$ measurement of the wire electrode displacement in combination with the simulation realized real-time measurement of the discharge reaction force and 3D wire behavior.
3) The results of the rough-cutting experiment showed that the discharge reaction force per single discharge increases as the cutting depth increases. This is probably because the pressure of the bubble increases in the narrow space.

4) It was confirmed from the measurement using the optical sensor that the wire is bent and vibrated not only due to the discharge reaction force and collision but also due to the influence of the fluid flow.

\section{Acknowledgement}

This research was supported by the Grant-in-Aid from JSPS (18H01346). The authors are grateful to Sodick Co. Ltd. for their kind support in conducting the experiments.

\section{References}

1) S. Hinduja, M. Kunieda: Modelling of ECM and EDM Processes, Annals of the CIRP, 62, 2, (2013) 775-797.

2) F. Han, M. Kunieda, T. Sendai, Y. Imai: High Precision Simulation of WEDM Using Parametric Programming, Annals of the CIRP, 51, 1, (2002) 165-168.

3) S. Tomura, M. Kunieda: Analysis of Electromagnetic Force in WireEDM, Precision Engineering, 33, 3, (2009) 255-262.

4) X. Yue, X. Yang, M. Kunieda: Comparison of Electrical Discharge Machining Speed of Tool Electrodes with Different Thermo-physical Properties Related to Ease of Boiling, CIRP Conference on Electro Physical and Chemical Machining, 19, 1, (2018) 138-143.

5) A. Okada, Y. Uno, S. Onoda, S. Habib: Computational fluid dynamics analysis of working fluid flow and debris movement in wire EDMed kerf, Annals of the CIRP, 58, 1, (2009) 209-212.

6) A. Okada, T. Konishi, Y. Okamoto, H. Kurihara: Wire breakage and deflection caused by nozzle jet flushing in wire EDM, Annals of the CIRP, 64, 1, (2015) 233-236.

7) M. Nishikawa, M. Kunieda: Prediction of Wire-EDMed Surface by In-Process Measurement of Wire Electrode Behavior, J JSPE, 75, 9, (2009) 1078-1082. 